PROCEEDINGS OF THE

AMERICAN MATHEMATICAL SOCIETY

Volume 128, Number 2, Pages 611-619

S 0002-9939(99)05002-9

Article electronically published on July 28, 1999

\title{
AN EXTENSION OF A THEOREM OF NICOLAESCU ON SPECTRAL FLOW AND THE MASLOV INDEX
}

\author{
MARK DANIEL
}

(Communicated by Ronald A. Fintushel)

\begin{abstract}
In this paper we extend a theorem of Nicolaescu on spectral flow and the Maslov index. We do this by studying the manifold of Lagrangian subspaces of a symplectic Hilbert space that are Fredholm with respect to a given Lagrangian $L_{0}$. In particular, we consider the neighborhoods in this manifold of Lagrangians which intersect $L_{0}$ nontrivially.
\end{abstract}

\section{INTRODUCTION}

Let $M$ be a smooth, closed, oriented, odd dimensional Riemannian manifold which is split along a hypersurface $\Sigma$. So $M=M_{1} \cup_{\Sigma} M_{2}$ where $M_{1}$ and $M_{2}$ are smooth, oriented, odd dimensional Riemannian manifolds with boundaries diffeomorphic to $\Sigma$. Let $D$ be a Dirac operator defined on a vector bundle $E$ over $M$. The symbol of $D$ defines a complex structure $J$ on the Hilbert space $H=L^{2}\left(\left.E\right|_{\Sigma}\right)$, which can be used to give $H$ the structure of a symplectic Hilbert space. The operator $D$ also determines Lagrangian subspaces $\Lambda_{1}(D)$ and $\Lambda_{2}(D)$ of $H$, the Cauchy data spaces. Roughly, $\Lambda_{i}(D)$ consists of the restrictions to the boundary of elements of ker $\left.D\right|_{M_{i}}$. In [7] Nicolaescu proves that if $D(t)(t \in I)$ is a path of Dirac operators such that $D(0)$ and $D(1)$ are invertible, then the spectral flow of the path $D(t)$ equals the Maslov index of the paths of Lagrangians $\Lambda_{1}(D(t))$ and $\Lambda_{2}(D(t))$. In this paper, we extend this result to the case where $D(0)$ and/or $D(1)$ have nontrivial kernel.

Treating the case where the endpoints have nontrivial kernel is a matter of reconciling various conventions for computing the spectral flow and the Maslov index. In the case of the spectral flow, it is customary to perturb the path of operators to eliminate the kernel at the endpoints. There is no canonical way of doing this, so one must make a choice. For example, one can replace the path of operators $A(t)$ by $A(t)+\epsilon I d$. This shifts the entire spectrum of each $A(t)$ up by $\epsilon$ so that elements in the kernel of $A(t)$ now have eigenvalue $\epsilon$ (with respect to $A(t)+\epsilon I d$ ). If $\epsilon$ is sufficiently small, then no other eigenvectors of $A(0)$ or $A(1)$ will be shifted into the kernel. Of course this choice of perturbation is a convention and other reasonable choices occur in the literature, but for this paper it is appropriate. We will refer to it as the spectral flow convention.

Received by the editors January 20, 1998 and, in revised form, April 7, 1998.

1991 Mathematics Subject Classification. Primary 57M99; Secondary 53C15, 58G25.

Key words and phrases. Spectral flow, Maslov index, Lagrangian subspace.

(C)1999 American Mathematical Society 
A similar situation arises when one tries to define the Maslov index of a pair of paths of Lagrangians $L_{1}(t)$ and $L_{2}(t)$ that intersect nontrivially at the endpoints. In this case, the choice of perturbation is suggested by the following observation. If $K_{1}$ and $K_{2}$ are a pair of Lagrangians (Fredholm in the infinite dimensional case), then

$$
e^{t J} K_{1} \cap K_{2}=0
$$

for all sufficiently small $t$. See [3] for a proof. Thus we define

$$
\mu\left(L_{1}, L_{2}\right)=\mu\left(e^{\epsilon J} L_{1}, L_{2}\right)
$$

where $\epsilon>0$ is chosen sufficiently small. Again, this is a matter of convention, but in this case it seems to be the only choice apparent in the literature. We will refer to this as the Maslov index convention.

To extend the theorem of Nicolaescu we will show that these perturbations can be reconciled. The theorem is extended by the following string of equalities:

$$
\begin{aligned}
S F(D) & =S F(D+\epsilon I d) & & \text { (spectral flow convention) } \\
& =\mu\left(\Lambda_{1}(D+\epsilon I d), \Lambda_{2}(D+\epsilon I d)\right) & & \text { (Nicolaescu's theorem) } \\
& =\mu\left(e^{\epsilon J} \Lambda_{1}(D), \Lambda_{2}(D)\right) & & \text { (proved in this paper) } \\
& =\mu\left(\Lambda_{1}(D), \Lambda_{2}(D)\right) & & \text { (Maslov index convention). }
\end{aligned}
$$

This paper is organized as follows. In Section 2 we show that locally, a pair of paths of Lagrangians determines a finite dimensional vector space and a path of self adjoint operators whose spectral flow equals the Maslov index. This allows us to show that paths of Lagrangians restricted to sufficiently small neighborhoods have bounded Maslov index and more importantly that paths which attain the bound have endpoints in prescribed path components of the complement of the Maslov cycle. In Section 3 we show that paths corresponding to the Maslov index convention described above attain the bound for the Maslov index in a suitable neighborhood. In light of the work of Section 2, this shows that their endpoints must lie in specified components of the complement of the Maslov cycle. In Section 4 we complete the proof appealing to homotopy invariance of the Maslov index.

We close this introduction with a few remarks. In cases where one wants to compute the spectral flow (e.g. Floer homology) it is often the case that the path of Dirac operators in question has nontrivial kernel at the endpoints. The work of this paper extends Nicolaescu's result to handle these situations. Moreover, this extension can be used to obtain a splitting formula for spectral flow similar to that of Cappell, Lee and Miller [1], [2]. This is carried out in [4].

\section{LOCAL COMPUTATIONS}

We begin by summarizing the standard coordinate charts for Lagrangians and the definition of the Maslov index. Details can be found in [4], [7]. Let $H$ be a symplectic Hilbert space. More specifically, $H$ is a real Hilbert space with inner product denoted by $\langle\cdot, \cdot\rangle$ and an operator $J \in O(H)$ which is skew adjoint and $J^{2}=-I d$. A symplectic form on $H$ is then defined by

$$
\omega(x, y)=\langle J x, y\rangle \text {. }
$$

A subspace $L$ of $H$ is called Lagrangian if $L^{\perp}=J L$. The collection of all Lagrangian subspaces of $H(\mathfrak{L}(H))$ is a smooth Banach manifold. For any $L_{0} \in \mathfrak{L}(H)$, the collection of all Lagrangians that are Fredholm with respect to $L_{0}$ is an open 
submanifold of $\mathfrak{L}(H)$ which we denote by $\mathfrak{L}_{F}\left(L_{0}\right)$. A convenient atlas for $\mathfrak{L}_{F}\left(L_{0}\right)$ is obtained as follows. Let $V$ be any finite dimensional subspace of $L_{0}$ and $W$ the orthogonal complement of $V$ in $L_{0}$. Let $\mathfrak{S}(V \oplus J W)$ denote the self adjoint operators on $V \oplus J W$ and define

$$
\Phi_{V}: \mathfrak{S}(V \oplus J W) \rightarrow \mathfrak{L}_{F}\left(L_{0}\right)
$$

by

$$
\Phi_{V}(A)=\text { the graph of } J A=\{x+J A x \mid x \in V \oplus J W\} .
$$

Then $\Phi_{V}$ is a homeomorphism ${ }^{1}$ and we denote its image by $O_{V}$. In fact $O_{V}$ may be described (without reference to $\Phi_{V}$ ) as the collection of all Lagrangians which are Fredholm with respect to $J V \oplus W$ and intersect it only at 0 . The collection of all pairs $\left(O_{V}, \Phi_{V}\right)$ as $V$ ranges over all finite dimensional subspaces of $L_{0}$ (along with their images) constitutes an atlas for $\mathfrak{L}_{F}\left(L_{0}\right)$. We refer to $\left(O_{V}, \Phi_{V}\right)$ as a standard coordinate chart.

The Maslov cycle of $\mathfrak{L}_{F}\left(L_{0}\right)$ is the subset

$$
\mathfrak{M}\left(L_{0}\right)=\left\{L \in \mathfrak{L}_{F}\left(L_{0}\right) \mid L \cap L_{0} \neq 0\right\} .
$$

It is a stratified (by dimension of $L \cap L_{0}$ ) subvariety. We denote the complement of $\mathfrak{M}\left(L_{0}\right)$ by $\mathfrak{L}_{F}\left(L_{0}\right)^{0}$. If we let

$$
\mathfrak{M}^{k}\left(L_{0}\right)=\left\{L \in \mathfrak{L}_{F}\left(L_{0}\right) \mid \operatorname{dim}\left(L \cap L_{0}\right)=k\right\},
$$

then it can be shown that the codimension of $\mathfrak{M}^{k}\left(L_{0}\right)$ is $k(k+1) / 2$. Moreover, the vector field $\nu$ associated with the flow

$$
(L, t) \mapsto e^{t J} L
$$

orients the normal bundle of $\mathfrak{M}^{1}\left(L_{0}\right)$. Consequently, if

$$
\gamma:(I, \partial I) \rightarrow\left(\mathfrak{L}_{F}\left(L_{0}\right), \mathfrak{L}_{F}\left(L_{0}\right)^{0}\right),
$$

then the intersection number of $\gamma$ with $\mathfrak{M}^{1}\left(L_{0}\right)$ is well defined and depends only on the homotopy class of $\gamma$ (as a map of pairs). This intersection number is called the Maslov index of $\gamma$ and denoted $\mu(\gamma)$.

Now, if $L_{1}$ and $L_{2}$ are paths in $\mathfrak{L}(H)$ such that $L_{1}(t)$ and $L_{2}(t)$ are Fredholm for each $t \in I$, we can find a path $T$ of linear isomorphisms of $H$ which commute with $J$ (symplectic isomorphisms) such that $T(t) L_{2}(t)=L_{2}(0)$ ([4], [6]). If we let $\gamma(t)=T(t) L_{1}(t)$, then $\gamma: I \rightarrow \mathfrak{L}_{F}\left(L_{2}(0)\right)$. Furthermore, if $L_{1}(i) \cap L_{2}(i)=0$ for $i=0,1$, then $\gamma:(I, \partial I) \rightarrow\left(\mathfrak{L}_{F}\left(L_{2}(0)\right), \mathfrak{L}_{F}^{0}\left(L_{2}(0)\right)\right)$ and thus $\mu(\gamma)$ is well defined. It can be shown ([4], [7]) that $\mu(\gamma)$ does not depend on the particular path $T$ which fixes $L_{2}$. Thus we may define the Maslov index of $L_{1}$ and $L_{2}$ by

$$
\mu\left(L_{1}, L_{2}\right)=\mu(\gamma)
$$

So we have two versions of the Maslov index. We will work primarily with the first version but apply the results (or more specifically their implications) to the latter version trusting the reader to make the translation.

Now, for any finite dimensional subspace $V$ of $L_{0} \in \mathfrak{L}(H)$, let $\left(O_{V}, \Phi_{V}\right)$ be the corresponding standard coordinate chart for $\mathfrak{L}_{F}\left(L_{0}\right)$. We now define a map $\Psi_{V}$ : $O_{V} \rightarrow \mathfrak{S}(V)$ by $\Psi_{V}(L)=\left.\pi_{V} \Phi_{V}^{-1}(L)\right|_{V}$ where $\pi_{V}$ denotes orthogonal projection onto $V$.

\footnotetext{
${ }^{1} \mathfrak{S}(V \oplus J W)$ is given the norm topology and $\mathfrak{L}_{F}\left(L_{0}\right)$ is given the gap topology. See [4] or [7] for details.
} 
Lemma 2.1. If $L \in O_{V}$, then $L \cap L_{0}$ is isomorphic to the kernel of $\Psi_{V}(L)$.

Proof. Let $A=\Phi_{V}^{-1}(L)$. If $x \in L \cap L_{0}$, then there exists $v \in V$ and $w \in W$ such that

$$
(I d+J A)(v+J w)=x
$$

because $L$ is the graph of $J A$. So

$$
v+J A v+J w+J A J w \in L_{0} .
$$

Since $L_{0}=V \oplus W$ and $L_{0}$ is Lagrangian, it follows that $J w=0$ and consequently $w=0$. Thus

$$
v+J A v \in L_{0} .
$$

Now, $J A v \in J V \oplus W$, so (2.1) implies that

$$
\pi_{J V} J A v=0 .
$$

It follows that

$$
J \pi_{J V} J A v=0 .
$$

Now, since $J \pi_{J V} J=-\pi_{V}$ we obtain

$$
\pi_{V} A v=0
$$

so $v \in \operatorname{ker}\left(\pi_{V} A\right)$. Observe that $\pi_{V} A=\Psi_{V}(L)$ and thus $(I d+J A)^{-1}$ maps $L \cap L_{0}$ injectively into $\operatorname{ker}\left(\Psi_{V}(L)\right)$.

Conversely, if $\Psi_{V}(L) v=0$, then

$$
(I d+J A) v \in L_{0} .
$$

This completes the proof of the lemma.

The previous lemma suggests that there might be a connection between the Maslov index of paths in $O_{V}$ and the spectral flow of their images under $\Psi_{V}$ in $\mathfrak{S}(V)$, because the Maslov index counts jumps in the dimension of $L \cap L_{0}$ while the spectral flow counts jumps in the dimension of the kernel of the given operator. We need only show that the signs of the contributions match. In the next lemma we use $O_{V}^{0}$ to denote the intersection of $O_{V}$ with the complement of $\mathfrak{M}\left(L_{0}\right)$.

Lemma 2.2. Let $\gamma:(I, \partial I) \rightarrow\left(O_{V}, O_{V}^{0}\right)$. Then

$$
\mu(\gamma)=S F\left(\Psi_{V} \circ \gamma\right)
$$

Proof. Both $\mu(\gamma)$ and $S F\left(\Psi_{V} \circ \gamma\right)$ depend only on the homotopy class of $\gamma$. Moreover, $\gamma$ is homotopic (rel endpoints) to a smooth $\tilde{\gamma}$ with the property that $\tilde{\gamma}(t) \cap L_{0}=$ 0 except at a discrete subset $\left\{t_{1}, \ldots, t_{n}\right\} \subset I$ at which $\tilde{\gamma}\left(t_{i}\right) \cap L_{0}$ is one dimensional. Thus, we may suppose $\gamma$ itself has this property. For such a path, the Maslov index is a sum of \pm 1 for each $t \in\left\{t_{1}, \ldots, t_{n}\right\}$. The sign of the contribution is determined by comparing $\gamma^{\prime}\left(t_{i}\right)$ with the vector field $\nu$ generated by the flow

$$
(s, L) \mapsto e^{s J} L .
$$

A straightforward computation (carried out in [4] for example) shows that this sign is equivalent to

$$
-\operatorname{sign}\left(\omega\left(v^{\prime}\left(t_{i}\right), v\left(t_{i}\right)\right)\right)
$$


where $v(t)$ is any smooth path of vectors in $\gamma(t)$ such that $v\left(t_{i}\right)$ spans the one dimensional subspace $\gamma\left(t_{i}\right) \cap L_{0}$.

We focus our attention on a particular $t_{i} \in\left\{t_{1}, \ldots, t_{n}\right\}$. Let $A(t)=\Psi_{V}(\gamma(t))$. By Lemma 2.1, 0 is a multiplicity one eigenvalue of $A\left(t_{i}\right)$. Let $\lambda(t)$ parametrize this eigenvalue and let $\phi(t)$ be the corresponding parametrized eigenvector (of unit length). The existence of $\lambda(t)$ and $\phi(t)$ depends on the fact that the eigenvalue in question is isolated and has multiplicity one [5]. Now, let

$$
v(t)=(I d+J A(t)) \phi(t)
$$

so that $v(t) \in \gamma(t)$ and $v\left(t_{i}\right)$ spans $\gamma\left(t_{i}\right) \cap L_{0}$.

Now,

$$
\lambda(t)=\langle A(t) \phi(t), \phi(t)\rangle .
$$

Thus

$$
\lambda^{\prime}\left(t_{i}\right)=\left\langle A^{\prime}\left(t_{i}\right) \phi\left(t_{i}\right), \phi\left(t_{i}\right)\right\rangle+\left\langle A\left(t_{i}\right) \phi^{\prime}\left(t_{i}\right), \phi\left(t_{i}\right)\right\rangle
$$

because $A\left(t_{i}\right) \phi\left(t_{i}\right)=0$. The sign of this expression is the contribution to the spectral flow of $A(t)$ at $t_{i}$.

On the other hand, we can compute

$$
\begin{aligned}
-\omega\left(v^{\prime}\left(t_{i}\right), v\left(t_{i}\right)\right) & =-\left\langle J\left(\left(I d+J A\left(t_{i}\right)\right) \phi\left(t_{i}\right)\right)^{\prime},\left(I d+J A\left(t_{i}\right)\right) \phi\left(t_{i}\right)\right\rangle \\
& =-\left\langle-A^{\prime}\left(t_{i}\right) \phi\left(t_{i}\right)+J\left(I d+J A\left(t_{i}\right)\right) \phi^{\prime}\left(t_{i}\right), \phi\left(t_{i}\right)\right\rangle \\
& =\left\langle A^{\prime}\left(t_{i}\right) \phi\left(t_{i}\right), \phi\left(t_{i}\right)\right\rangle+\left\langle J \phi^{\prime}\left(t_{i}\right), \phi\left(t_{i}\right)\right\rangle-\left\langle A\left(t_{i}\right) \phi^{\prime}\left(t_{i}\right), \phi\left(t_{i}\right)\right\rangle \\
& =\left(\left\langle A^{\prime}\left(t_{i}\right) \phi\left(t_{i}\right), \phi\left(t_{i}\right)\right\rangle+\left\langle A\left(t_{i}\right) \phi^{\prime}\left(t_{i}\right), \phi\left(t_{i}\right)\right\rangle\right)
\end{aligned}
$$

where the term $\left\langle J \phi^{\prime}\left(t_{i}\right), \phi\left(t_{i}\right)\right\rangle=0$ because $J \phi^{\prime}\left(t_{i}\right) \in J V$ and $\phi\left(t_{i}\right) \in V(J V$ is orthogonal to $V)$.

Comparing (2.2) and (2.3) we see that the contributions to the Maslov index and the spectral flow are the same at $t_{i}$. This completes the proof of the lemma.

We obtain as an immediate corollary:

Corollary 2.3. If $\operatorname{dim}(V)=n$ and $\gamma:(I, \partial I) \rightarrow\left(O_{V}, O_{V}^{0}\right)$, then

$$
-n \leq \mu(\gamma) \leq n
$$

Proof. This follows from the corresponding bound on the spectral flow of a path of operators on a finite dimensional vector space. Recall that in the finite dimensional context, the spectral flow of a path of operators is one half the difference of the signatures at the endpoints.

We continue to explore the implications of this relationship between Maslov index and spectral flow. Let $\mathfrak{S}(V)^{0}$ denote the subset of $\mathfrak{S}(V)$ consisting of invertible operators. $\mathfrak{S}(V)^{0}$ has exactly $n+1$ path components corresponding to the number of positive eigenvalues of a given $A$. Moreover, the map $\Psi_{V}$ establishes a 1:1 correspondence between the path components of $\mathfrak{S}(V)^{0}$ and those of $O_{V}^{0}$.

Now, if $\gamma:(I, \partial I) \rightarrow\left(\mathfrak{S}(V), \mathfrak{S}(V)^{0}\right)$ has spectral flow $n$, then it must be that $\gamma(0)$ has all negative eigenvalues and $\gamma(1)$ has all positive eigenvalues. This establishes the following corollary.

Corollary 2.4. There exist path components $\Gamma_{0}$ and $\Gamma_{1}$ of $O_{V}^{0}$ such that any $\gamma$ : $(I, \partial I) \rightarrow\left(O_{V}, O_{V}^{0}\right)$ has Maslov index $n$ if and only if $\gamma(0) \in \Gamma_{0}$ and $\gamma(1) \in \Gamma_{1}$. 
In fact, homotopy invariance of the Maslov index implies that for any $\gamma$ : $(I, \partial I) \rightarrow\left(O_{V}, O_{V}^{0}\right), \mu(\gamma)$ depends only on the endpoints of the path. The preceding corollary shows that in the case where the Maslov index takes on its maximum (or minimum) value, a converse to this statement is true.

\section{The Canonical Flow yields maximal Maslov indeX}

In the previous section we showed that locally, paths having maximal Maslov index have prescribed endpoints. In this section we will show that the path

$$
t \mapsto e^{t J} L
$$

has maximal Maslov index. Intuitively, this is because the vector field associated with this flow determines the positive orientation of the Maslov cycle. Consequently, each nontrivial intersection along such a path contributes positively to the Maslov index.

Let $H$ be a symplectic Hilbert space and $L_{0} \in \mathfrak{L}(H)$. Suppose $L \in \mathfrak{L}_{F}\left(L_{0}\right)$ such that $\operatorname{dim}\left(L \cap L_{0}\right)=n$. Finally, let

$$
\gamma(t)=e^{t J} L .
$$

Lemma 3.1. For all sufficiently small $\epsilon>0, \gamma(-\epsilon) \cap L_{0}=\gamma(\epsilon) \cap L_{0}=0$ and $\mu(\gamma)=n$.

Proof. The fact that $\gamma(-\epsilon) \cap L_{0}=\gamma(\epsilon) \cap L_{0}=0$ for sufficiently small $\epsilon$ is proved in [3]. To prove the rest of the conclusion we will use Lemma 2.2. Let $V=L_{0} \cap L$ and let $W$ be the orthogonal complement of $V$ in $L_{0}$. Let $A(t)=\Phi_{V}^{-1}(\gamma(t))$. We will denote $A(0)$ by $A_{0}$. From the definition of $\gamma(t)$ we must have

$$
e^{t J}\left(I d+J A_{0}\right)=I d+J A(t) .
$$

Thus

$$
A(t)=-J e^{t J}-J e^{t J} J A_{0}+J .
$$

This implies that

$$
\Psi_{V}(\gamma(t))=-\left.\pi_{V}\left(J e^{t J}+J e^{t J} J A_{0}-J\right)\right|_{V} .
$$

Now, $\left.\pi_{V} J\right|_{V}=0$ (because $J V \perp V$ ) and $\left.A_{0}\right|_{V}=0$ (because $\operatorname{dim}\left(\gamma(0) \cap L_{0}\right)=n=$ $\operatorname{dim}(V))$. Thus

$$
\Psi_{V}(\gamma(t))=-\left.\pi_{V} J e^{t J}\right|_{V}
$$

It turns out that we can compute the right hand side explicitly. For any $v \in V$, we have

$$
\begin{aligned}
-\pi_{V} J e^{t J} v & =-\pi_{V} J(\cos t v+\sin t J v) \\
& =\sin t v
\end{aligned}
$$

Thus

$$
\Psi_{V}(\gamma(t))=\sin t I d
$$

and the conclusions of this lemma follow immediately from Lemmas 2.1 and 2.2.

The significance of this lemma is that combined with Corollary 2.4, it implies that for all sufficiently small $\epsilon>0, e^{t J} L$ is in the path component $\Gamma_{1}$ of $O_{V}$. 


\section{Conclusion}

In this section we prove the missing step in the string of equalities (1.1-1.4) presented in the introduction. Namely, we show that

$$
\mu\left(\Lambda_{1}(D+\epsilon I d), \Lambda_{2}(D+\epsilon I d)\right)=\mu\left(e^{\epsilon J} \Lambda_{1}(D), \Lambda_{2}(D)\right)
$$

thereby reconciling the spectral flow convention with the Maslov index convention. Recall that $D$ is a path of Dirac operators on some bundle $E$ over a split manifold $M=M_{1} \cup_{\Sigma} M_{2}$.

It is convenient to isolate the following elementary result rather than include its proof in the proof of Theorem 4.2 below.

Lemma 4.1. Suppose $\gamma_{0}, \gamma_{1}:(I, \partial I) \rightarrow(X, Y)(Y \subset X$ topological spaces $)$ are homotopic as maps from $I$ to $X$ (freely homotopic) by a homotopy $H: I \times I \rightarrow X$ $\left(H(x, 0)=\gamma_{0}(x), H(x, 1)=\gamma_{1}(x)\right)$. If $H(0, \cdot)$ and $H(1, \cdot)$ are each homotopic (rel endpoints) to paths contained in $Y$, then $\gamma_{0}$ and $\gamma_{1}$ are homotopic as maps from $(I, \partial I)$ to $(X, Y)$, i.e. there exists a homotopy $\tilde{H}$ as above such that $\tilde{H}(0, y) \in Y$ and $\tilde{H}(1, y) \in Y$ for all $y \in I$.

Proof. Let $K_{0}$ be a homotopy (rel endpoints) from a path in $Y$ to $H(0, \cdot)$, so $K_{0}(1, \cdot)=H(0, \cdot)$ and $K_{0}(0, \cdot)$ is a path in $Y$. Similarly, let $K_{1}$ be a homotopy (rel endpoints) from $H(1, \cdot)$ to a path in $Y$; thus $K_{1}(1, \cdot)=H(1, \cdot)$ and $K_{1}(0, \cdot)$ is a path in $Y$. A formula for a homotopy $\tilde{H}$ satisfying the properties stated above is

$$
\tilde{H}(t, s)= \begin{cases}K_{0}\left(\frac{2 t}{s}, s\right), & 0<s \leq \frac{1}{2} t<\frac{s}{2}, \\ K_{0}(0,0), & s=0 t=0, \\ K_{0}\left(\frac{2 t}{1-s}, s\right), & \frac{1}{2}<s<1 t<\frac{1-s}{2}, \\ K_{0}(0,1), & s=1 t=0, \\ H\left(\frac{2 t-s}{2-2 s}, s\right), & s \leq \frac{1}{2} \frac{s}{2} \leq t \leq \frac{2-s}{2}, \\ H\left(\frac{2 t+s-1}{2 s}, s\right), & \frac{1}{2}<s \frac{1-s}{2} \leq t \leq \frac{1+s}{2}, \\ K_{1}\left(\frac{2 t+s-2}{s}, s\right), & 0<s \leq \frac{1}{2} \frac{2-s}{2}<t, \\ K_{1}(1,0), & s=0 t=1, \\ K_{1}\left(\frac{2 t-s-1}{1-s}, s\right), & \frac{1}{2}<s<1 \frac{s+1}{2}<t, \\ K_{1}(1,1), & s=1 t=1 .\end{cases}
$$

Now we are ready to prove the missing step (1.3) in the string of equalities in the introduction.

Theorem 4.2. Let $D(t)$ ( $t \in I)$ be a path of Dirac operators on a bundle $E$ over a closed, oriented, odd dimensional Riemannian manifold which splits as $M=$ $M_{1} \cup_{\Sigma} M_{2}$. For all sufficiently small $\epsilon, \delta>0$

$$
\mu\left(e^{\epsilon J} \Lambda_{1}(D), \Lambda_{2}(D)\right)=\mu\left(\Lambda_{1}(D+\delta I d), \Lambda_{2}(D+\delta I d)\right) .
$$

Proof. First choose $T_{1}(t)(t \in I)$ a path of symplectic isomorphisms of $H=L^{2}\left(\left.E\right|_{\Sigma}\right)$ such that

$$
T_{1}(t) \Lambda_{2}(D(t))=\Lambda_{2}(D(0))
$$


and $T_{1}(0)=I d$. Next choose $T_{2}(t, s)(t \in I, s \in[-\delta, \delta])$ a family of symplectic isomorphisms of $H$ such that

$$
T_{2}(t, s) \Lambda_{2}(D(t)+s I d)=\Lambda_{2}(D(0)) .
$$

The construction of $T_{1}$ and $T_{2}$ is detailed in [6] or [4].

Now, let $S(t, s)=T_{2}(t, 0)^{-1} T_{2}(t, s)$ and observe that

$$
S(t, s) \Lambda_{2}(D(t)+s I d)=\Lambda_{2}(D(t))
$$

and $S(t, 0)=I d$. To simplify notation, let

$$
\begin{aligned}
L_{0} & =\Lambda_{2}(D(0)), \\
L_{1}(t) & =T_{1}(t) e^{\epsilon J} \Lambda_{1}(D(t)), \\
L_{2}(t) & =T_{1}(t) S(t, \delta) \Lambda_{1}(D(t)+\delta I d) .
\end{aligned}
$$

Then to prove the lemma, it suffices to show that

$$
\mu\left(L_{1}, L_{0}\right)=\mu\left(L_{2}, L_{0}\right),
$$

and to do this, it suffices to show that $L_{1}$ and $L_{2}$ are homotopic as maps from $(I, \partial I)$ to $\left(\mathfrak{L}_{F}\left(L_{0}\right), \mathfrak{L}_{F}\left(L_{0}\right)-\mathfrak{M}\left(L_{0}\right)\right)$. by

First, observe that $L_{1}$ and $L_{2}$ are freely homotopic via $H: I \times[-\delta, \epsilon] \rightarrow \mathfrak{L}_{F}\left(L_{0}\right)$

$$
H(t, s)= \begin{cases}T_{1}(t) e^{s J} \Lambda_{1}(D(t)), & s \geq 0, \\ T_{1}(t) S(t,-s) \Lambda_{1}(D(t)-s I d), & s<0,\end{cases}
$$

because $S(t, 0)=I d$.

Now we examine the path traced out by the left endpoint of the homotopy. Let $\gamma(s)=H(0, s)$. Suppose that $\gamma(0) \in \mathfrak{M}^{n}\left(L_{0}\right)$, i.e. $\operatorname{dim}\left(\Lambda_{1}(D(0)) \cap \Lambda_{2}(D(0))\right)=n$ so $D(0)$ has nontrivial kernel. Let $V=\Lambda_{1}(D(0)) \cap \Lambda_{2}(D(0))$ and define $O_{V}$ and $\Gamma_{1}$ as in Corollary 2.4. We claim that $\gamma(s) \in \Gamma_{1}$ for all $s \neq 0$. To see this, first observe that if $\delta$ is sufficiently small, the spectral flow of

$$
s \mapsto D(0)+s I d
$$

is precisely $n=\operatorname{dim}(V)$ and $\operatorname{ker}(D(0) \pm \delta I d)=0$. Thus we may apply Nicolaescu's theorem to conclude that

$$
\mu\left(\Lambda_{1}(D(0)+s I d), \Lambda_{2}(D(0)+s I d)\right)=n .
$$

This is equivalent to

$$
\mu\left(S(0, s) \Lambda_{1}(D(0)+s I d), \Lambda_{2}(D(0))\right)=n .
$$

Consequently, by Corollary 2.4 we conclude that $S(0, s) \Lambda_{1}(D(0)+s I d) \in \Gamma_{1}$ for $s \in(0, \delta)$. Equivalently, $\gamma(s) \in \Gamma_{1}$ for $s \in[-\delta, 0)$. For $\gamma(s)$ where $s \in(0, \epsilon]$, observe that (Lemma 3.1)

$$
\mu\left(e^{s J} \Lambda_{1}(D(0)), \Lambda_{2}(D(0))\right)=n
$$

and apply Corollary 2.4 as above.

Now, $\Gamma_{1}$ is path connected, so there exists a path $\tilde{\gamma}$ between $\gamma(-\delta)$ and $\gamma(\epsilon)$ which lies entirely in $O_{V}$. Moreover, $\Gamma_{1} \cup\left(\mathfrak{M}^{n}\left(L_{0}\right) \cap O_{V}\right)$ is contractible. To see this, note that in the standard coordinates $\left(O_{V}, \Phi_{V}\right)$, $\Phi_{V}^{-1}\left(\Gamma_{1} \cup\left(\mathfrak{M}^{n}\left(L_{0}\right) \cap O_{V}\right)\right)=\left\{A \in \mathfrak{S}(V \oplus J W) \mid\right.$ the spectrum of $\Psi_{V}(A)$ is positive

$$
\text { or } \left.\Psi_{V}(A)=0\right\}
$$


and this set is contractible to 0 via the map

$$
(s, A) \mapsto(1-s) A
$$

for $s \in[0,1]$. Contractibility of this set implies that $\gamma$ is homotopic rel endpoints to $\tilde{\gamma}$.

So, we have shown that $L_{1}$ and $L_{2}$ are homotopic via a homotopy $H$ such that $H(0, \cdot)$ is homotopic rel endpoints to a path which misses $\mathfrak{M}\left(L_{0}\right)$. Similarly we can show that $H(1, \cdot)$ is homotopic rel endpoints to a path which misses $\mathfrak{M}\left(L_{0}\right)$. Thus we may apply Lemma 4.1 to conclude that $L_{1}$ and $L_{2}$ are homotopic as maps $(I, \partial I)$ to $\left(\mathfrak{L}_{F}\left(L_{0}\right), \mathfrak{L}_{F}\left(L_{0}\right)-\mathfrak{M}\left(L_{0}\right)\right)$. This completes the proof of the lemma.

We close this paper with a statement of the extension of the theorem of Nicolaescu implied by the above lemma.

Theorem 4.3. Let $D(t)$ be a smooth path of Dirac operators on a smooth, oriented, closed, odd dimensional Riemannian manifold $M$ which splits as $M=M_{1} \cup_{\Sigma} M_{2}$. Then

$$
S F(D)=\mu\left(\Lambda_{1}(D), \Lambda_{2}(D)\right)
$$

Proof. The case where $D(0)$ and $D(1)$ have trivial kernel is proved by Nicolaescu in [7]. The general case is obtained from that result by applying Theorem 4.2 and the string of equalities (1.1-1.4).

\section{REFERENCES}

1. S.Cappell, R.Lee, \& E.Miller, Self-adjoint elliptic operators and manifold decompositions, Part I: Low eigenmodes and stretching, Comm. Pure Appl. Math 49 (1996), 825-866. MR 97g:58162

2. S.Cappell, R.Lee, \& E.Miller, Self-adjoint elliptic operators and manifold decompositions, Part II: Spectral flow and Maslov index, Comm. Pure Appl. Math 49 (1996), 869-909. MR 97g:58163

3. S.Cappell, R.Lee, \& E.Miller, On the Maslov index, Comm. Pure Appl. Math. 47 (1994), 121-186. MR 95f:57045

4. A.M.Daniel, Maslov index, symplectic reduction in a symplectic Hilbert space and a splitting formula for spectral flow, Doctoral Dissertation, Indiana University, Bloomington, 1997.

5. T.Kato, Perturbation Theory for Linear Operators. Classics in Mathematics, Springer-Verlag, 1995. MR 96a: 47025

6. P.Kirk \& E.Klassen, Analytic deformations of the spectrum of a family of Dirac operators on an odd-dimensional manifold with boundary, Mem. Amer. Math. Soc. 124 (1996), number 592. MR 97d:58184

7. L.I.Nicolaescu, The Maslov index, the spectral flow and decompositions of manifolds, Duke Math J. 80 (1995), 485-533. MR 96k:58208

Applied Physics Operation, SAiC, McLean, Virginia 22102

E-mail address: amdaniel@ccf.nrl.navy.mil

Current address: Advanced Power Technologies, Inc., 1250 Twenty-Fourth St., NW, Suite 850, Washington, DC 20037

E-mail address: amdaniel@apti.com 